\title{
Infiltrating $\mathrm{CD}^{+}{ }^{+}$Are Associated with a Reduction in Peripheral $\mathrm{CD}_{14}{ }^{+} \mathrm{CD}^{++}{ }^{++}$Monocytes and Severe Forms of Lupus Nephritis
}

\author{
Anabel Barrera García, ${ }^{1}$ José A. Gómez-Puerta, ${ }^{1,2}$ Luis F. Arias, ${ }^{3}$ \\ Catalina Burbano, ${ }^{1}$ Mauricio Restrepo, ${ }^{2}$ Adriana L. Vanegas, ${ }^{2}$ Carlos H. Muñoz, ${ }^{2}$ \\ Mauricio Rojas, ${ }^{1}$ Luis A. González, ${ }^{2}$ and Gloria Vásquez ${ }^{1,2}$ \\ ${ }^{1}$ Grupo de Inmunología Celular e Inmunogenética, GICIG, Universidad de Antioquía, Medellín, Colombia \\ ${ }^{2}$ Grupo de Reumatología, Universidad de Antioquía, Medellín, Colombia \\ ${ }^{3}$ Grupo de Patología Renal y Trasplante, Universidad de Antioquía, Medellín, Colombia
}

Correspondence should be addressed to Gloria Vásquez; glomavas@gmail.com

Received 27 July 2016; Revised 27 October 2016; Accepted 30 October 2016

Academic Editor: Ricard Cervera

Copyright ( 2016 Anabel Barrera García et al. This is an open access article distributed under the Creative Commons Attribution License, which permits unrestricted use, distribution, and reproduction in any medium, provided the original work is properly cited.

\begin{abstract}
Our aim was to characterize glomerular monocytes (Mo) infiltration and to correlate them with peripheral circulating Mo subsets and severity of lupus nephritis (LN). Methods. We evaluated 48 LN biopsy samples from a referral hospital. Recognition of Mo cells was done using microscopic view and immunohistochemistry stain with CD14 and CD16. Based on the number of cells, we classified LN samples as low degree of diffuse infiltration ( $<5$ cells) and high degree of diffuse infiltration ( $\geq 5$ cells). Immunophenotyping of peripheral Mo subsets was done using flow cytometry. Results. Mean age was 34.0 \pm 11.7 years and the mean SLEDAI was 17.5 \pm 6.9 . The most common SLE manifestations were proteinuria (91\%) and hypocomplementemia (75\%). Severe LN was found in $70 \%$ of patients (Class III, 27\%; Class IV, 43\%). Severe LN patients and patients with higher grade of CD16 ${ }^{+}$infiltration had lower levels of nonclassical $\left(\mathrm{CD}_{1} 4^{+} \mathrm{CD} 16^{++}\right)$Mo in peripheral blood. Conclusions. Our results might suggest that those patients with more severe forms of $\mathrm{LN}$ had a higher grade of $\mathrm{CD} 14^{+} \mathrm{CD} 16^{+}$infiltration and lower peripheral levels of nonclassical $\left(\mathrm{CD} 14^{+} \mathrm{CD} 16^{++}\right) \mathrm{Mo}$ and might reflect a recruitment process in renal tissues. However, given the small sample, our results must be interpreted carefully.
\end{abstract}

\section{Introduction}

Systemic lupus erythematosus (SLE) is the most representative of autoimmune diseases being renal involvement one of the main features. Lupus nephritis (LN) is one of the hallmark features of SLE, seen in $40 \%-60 \%$ of patients at the moment of diagnosis, and is one of main causes of mortality among these patients [1]. Among 10 to $15 \%$ can develop an end stage renal disease during the follow-up and might require renal replacement therapies and/or kidney transplant $[2,3]$.

In the pathogenesis of SLE, innate immunity and immune complex had been widely evaluated but several cellular mechanisms have not been described in depth. Monocytes (Mo) are heterogeneous cells in their morphology, phenotype, and function. In humans, three different subpopulations of circulating Mo have been described. Those subsets are defined according to the expression of CD14 and CD16. In healthy subjects, $90 \%$ of $\mathrm{Mo}$ are $\mathrm{CD} 14^{++} \mathrm{CD}^{-} 6^{-}$(classic Mo); $4 \%$ have the phenotype $\mathrm{CD} 14^{++} \mathrm{CD} 16^{+}$(intermediate) and are also considered mature and can participate in the inflammatory response. The third subpopulation are $\mathrm{CD} 14^{+} \mathrm{CD} 16^{++}$ (nonclassic) that in comparison with other subpopulations of Mo are low producers of IL-10 [4].

Although the role of these subpopulations is controversial, it is well known that they can recognize immune complex through CD16, additionally can differentiate to diverse types of cells such as macrophages or dendritic cells (DC), and can polarize adaptive immune responses [5].

The main modifications of subpopulations of Mo cells have not been studied extensively in SLE patients. We 
described recently that $\mathrm{CD} 14^{+} \mathrm{CD} 16^{++}$Mo are decreased in patients with active SLE and display changes in the molecules associated with their functions [6]. Those findings may suggest a potential role of these subpopulations in the pathogenesis of SLE and ultimately in kidney tissue lesions in LN.

There are enough "in vitro" evidences that a subpopulation of CD16 can differentiate to DC under the presence of GM-CSF, TGF- $\beta$, and IL-10, but without complete maturation. In that way, nonclassic $\mathrm{CD} 16^{+}$Mo might play a role as an important immunomodulator for innate and adaptive response in SLE participating in tissue damage, even before its differentiation to macrophages [7-10].

Some previous studies have investigated some subpopulations of Mo in murine models [11], but there is limited information in humans $[12,13]$. This is explained in part for the differences between murine and human phagocytes, for the wide variability on Mo subpopulations, and for the lack of equivalence in translation studies among murine and humans models.

Pathogenic mechanisms of LN are not well understood. Some previous studies suggested that phagocytes $\mathrm{CD} 14^{+} \mathrm{CD} 16^{+}$might participate in glomerular infiltration, interact with immune complex, and regulate some phases of inflammatory response including the process of antigen presentation [14].

Given the previous information and the lack of studies in patients with $\mathrm{LN}$, our aim was to characterize glomerular Mo infiltration in kidney tissue and to correlate those infiltrates with peripheral blood levels of Mo, disease features, disease activity, and histological characteristics.

\section{Material and Methods}

2.1. Selection of Sample. We included 328 biopsy samples with diagnosis of LN by histopathology and immunofluorescence from clinical records of Pathology Department, Universidad de Antioquia. 241 out of 328 samples were done at Hospital San Vicente Fundación at Medellín, Colombia, from 2000 to 2011. Only those patients older than 18 years were included $(n=169)$. Finally, 48 tissue samples were classified as good enough to perform new analysis.

\subsection{Histopathological Studies and Immunohistochemistry.} Kidney biopsies were embedded in paraffin and sectioned at $4 \mu \mathrm{m}$; then those samples were deparaffinized in xylene and rehydrated using graded concentrations of ethanol. Samples were stained with hematoxylin and eosin and then were examined under a light microscope. Tissue histopathologic state was classified according to ISN/RPS criteria. Patients with proliferative LN were grouped such as severe LN. Activity and chronicity indices of the histological appearance also were assessed based on National Institutes of Health scores. One observer (LA) with no prior knowledge of the clinical course examined renal tissue to establish the diagnosis by using standard pathological methods. Patients with renal involvement at SLE onset or with less than 1 year of disease duration were classified as early LN.
Different antibodies were used including CD14: Thermo Scientific, Rockford, Illinois, USA, number MA5-11394, dilution 1: 40; CD16: Thermo Scientific, number MA5-11413, dilution 1: 200; and CD68: Dako, Carpinteria, USA, code M0876, dilution 1:200. Those monoclonal antibodies were incubated at room temperature during 60 minutes. Endogenous peroxidase was blocked with hydrogen peroxide. Antigen retrieval was done with heat (steam) and tris-buffer with ethylenediaminetetraacetic acid at $\mathrm{pH} 7.0$ for 30 minutes. The detection system used was based on polymer (Ultravision Quanto, Thermo Fisher Scientific, Fremont, CA, USA).

Recognition of Mo cells was done using microscopic view. Positive markers were quantified using the following parameters: If the stain was positive but with $<2$ cells together the stain was classified as "diffuse," and the diffuse infiltrate was scored as follows: high degree of diffuse $\geq 5$ cells or low degree of diffuse $<5$ cells. Whether quantification detects more than 2 cells together was classified as "aggregates." Based on the number of cell counts aggregates were classified as grade 1, less than 5 cell counts; grade 2, five to 10 cell counts; and grade 3 , more than 10 cell counts.

2.3. Immunophenotyping of Monocytes Subsets. Mo subpopulations were defined as three subsets based on previous reports in classic, $\left(\mathrm{CD} 14^{++} \mathrm{CD} 16^{-}\right)$, nonclassic $\left(\mathrm{CD} 14^{+} \mathrm{CD} 16^{++}\right)$, and intermediate $\left(\mathrm{CD} 14^{++} \mathrm{CD} 16^{+}\right) \mathrm{Mo}[15]$. Briefly, 25 microliters of EDTA-anticoagulated peripheral blood was stained with anti-CD14-PerCP, anti-CD16-FITC, and anti-HLA-DR-PE or the isotype controls. Samples were mixed and incubated for $20 \mathrm{~min}$ at room temperature in darkness. Red cells were lysed with $250 \mu \mathrm{L}$ of OptiLyse and analyzed using the FACS Canto ${ }^{\mathrm{TM}}$ II (Becton Dickinson, San Jose, CA). Manual counting was compared with absolute counting. All patients gave informed consent for study. This study was approved by the Ethics Committee of the Universidad de Antioquia Medical Research Institute.

2.4. Statistical Analysis. Several variables were dichotomized for the analysis. Patients with proliferative LN were grouped as severe LN. Additionally patients were classified according to disease duration (early LN versus non-early) and according to degree of $\mathrm{CD}^{+} 6^{+}$infiltration (low or high level) as we mentioned above. Values are represented as means. Differences in means in normally distributed variables were analyzed using the parametric $t$-test, and variables without a normal distribution were assessed using Wilcoxon's test. The analysis was made using the IBM SPSS v22 statistical package.

\section{Results}

A total of 48 Mestizo patients with biopsy proven LN were enrolled for the study, of which $87 \%$ were females with a mean disease duration of $2.91 \pm 5.6$ years. Fifty-six percent of patients had early onset LN. Median age for the whole group was $34.0 \pm 11.7$ years. Proteinuria was the most frequent renal feature (91\%), followed by hematuria (66\%) and sterile pyuria $(38 \%)$. The main clinical features are summarized in Table 1. Mean 24-hour proteinuria levels were $4.668 \pm$ $3906 \mathrm{mg}$ and mean glomerular filtration rate (GFR) was 
TABLE 1: General characteristics of 48 SLE patients with biopsy proven LN.

\begin{tabular}{lc}
\hline Clinical characteristics & \\
Mean age (years \pm SD) & $34.0 \pm 11.7$ \\
Gender (female) & $87 \%$ \\
Proteinuria & $43(91.5 \%)$ \\
Hematuria & $31(66.0 \%)$ \\
Arthritis & $21(44.7 \%)$ \\
Pyuria & $18(38.3 \%)$ \\
Urinary casts & $16(34.0 \%)$ \\
Pleuritis & $11(23.4 \%)$ \\
Alopecia & $9(19.1 \%)$ \\
Mouth ulcers & $9(19.1 \%)$ \\
Malar rash & $8(17.0 \%)$ \\
Pericarditis & $6(12.8 \%)$ \\
Vasculitis & $6(12.8 \%)$ \\
Fever & $5(10.6 \%)$ \\
Headache & $1(2.1 \%)$ \\
Seizures & $1(2.1 \%)$ \\
SLEDAI (SD) & $17.5 \pm 6.9$ \\
\hline Laboratory findings & \\
Hypocomplementemia (either C3 or C4) & $38(80.8 \%)$ \\
dsDNA antibodies & $27(57.4 \%)$ \\
Leukopenia & $8(17 \%)$ \\
Thrombocytopenia & $8(17 \%)$ \\
\hline LN classification & \\
Class I & $2(4.2 \%)$ \\
Class II & $6(12.5 \%)$ \\
Class III $I V$ & $13(27.0 \%)$ \\
Class V & $21(43.8 \%)$ \\
Mean activity index score (SD) & $6(12.5 \%)$ \\
Mean chronicity index score (SD) & $5.7 \pm 4.9$ \\
\hline
\end{tabular}

$74.2 \pm 40.6 \mathrm{~mL} / \mathrm{min}$. As expected, proteinuria levels were significantly higher in patients with severe LN forms than in patients without severe forms $(4.301 \pm 917$ versus $626 \pm$ $280 \mathrm{mg}, p=0.018$ ). No differences in GFR were found among groups.

The Mean Systemic Lupus Erythematosus Disease Activity Index (SLEDAI) score was $17.5 \pm 6.9$. We observed a high disease activity in most of the patients, in part explained by the specific weight of renal involvement (12 points).

3.1. Pathological Features. Proliferative glomerulonephritis (Class IV) was detected in $21(43.8 \%)$ patients, Class III was detected in 13 (27\%) of patients, and Class II and Class V LN were detected in six (12.5\%) patients each. Only two patients were classified as Class I LN. We used activity and chronicity index proposed by Rovin [14] as our guide for the description of histological findings. Most common findings for acute lesion were cellular proliferation $(83 \%)$ and karyorrhexis (25\%). Tubular interstitial infiltrates were found in $71 \%$ of the
TABLE 2: Histopathology characteristics in 48 patients with biopsy proven LN.

\begin{tabular}{lc}
\hline Glomerular findings & \\
Local necrosis & $9(18.7 \%)$ \\
Cellular proliferation & $40(83.3 \%)$ \\
Karyorrhexis & $12(25.0 \%)$ \\
Fibrinoid exudate & $1(2.1 \%)$ \\
Wire loop lesions & $9(18.7 \%)$ \\
Hyaline thrombosis & $6(12.5 \%)$ \\
Basal membrane thickening & $13(27.1 \%)$ \\
Fibrosis & $4(8.3 \%)$ \\
\hline Tubulointerstitial findings & \\
Inflammatory infiltrate & $34(70.8 \%)$ \\
Edema & $1(2.1 \%)$ \\
Fibrosis & $18(37.5 \%)$ \\
Tubular atrophy & $18(37.5 \%)$ \\
\hline Artery and arterioles & \\
Fibrinoid exudate & $1(2.1 \%)$ \\
Fibrinoid/platelet thrombus & $1(2.1 \%)$ \\
Necrosis & $1(2.1 \%)$ \\
Arterial sclerosis & $5(10.4 \%)$ \\
Arteriolar hyalinosis & $4(8.3 \%)$ \\
\hline
\end{tabular}

cases. Arterial and arteriole abnormalities were found in a minority of patients.

Chronic changes were less common reported in renal biopsies. Tubular and glomerular fibrosis were found in $37 \%$ and $8 \%$ of patients, respectively. Other chronic changes were tubular atrophy in $37 \%$ of patients, basal membrane thickening in $27 \%$ of patients, arterial sclerosis in $10.4 \%$, and arterial hyalinosis in $8.3 \%$ of patients. Histological findings are shown in Table 2.

We compared activity and chronicity indices scores among Class II and Class IV LN based on National Institutes of Health scores. Both acute and chronicity indices on kidney biopsies were significantly higher in Class IV LN than in Class III LN patients. Mean acute index was $4.1 \pm 1.7$ versus $9.6 \pm 4.4$, $p=0.001$, for Classes III and IV, respectively, and mean chronicity index was $0.8 \pm 2.0$ versus $1.9 \pm 2.0, p=0.032$, for Classes III and IV, respectively.

3.2. Characterization of Mo Infiltrates. Diffuse $\mathrm{CD} 16^{+}$cells were found in 25 biopsies and $\mathrm{CD} 14^{+}$cells in only sixteen biopsies. Representative pictures are shown in Figure 1 of the immunostainings for $\mathrm{CD}_{16}{ }^{+}$and CD14 cells.

Patients with higher degree of CD16 diffuse infiltrates had a higher prevalence of hypocomplementemia (100\% versus $0 \%, p=0.028$ ), higher but not significant levels of dsDNA antibodies, and higher disease activity (SLEDAI score $\geq$ 10). Five patients had $\mathrm{CD}^{+} 6^{+}$Mo aggregates. All patients who had grade 3 aggregates had Class IV LN in histologic classification. Grade 1 was observed in one patient with Class II and aggregates grade 2 was observed in one patient with Class III LN. With respect to $\mathrm{CD}_{1} 4^{+}$Mo cells, only 


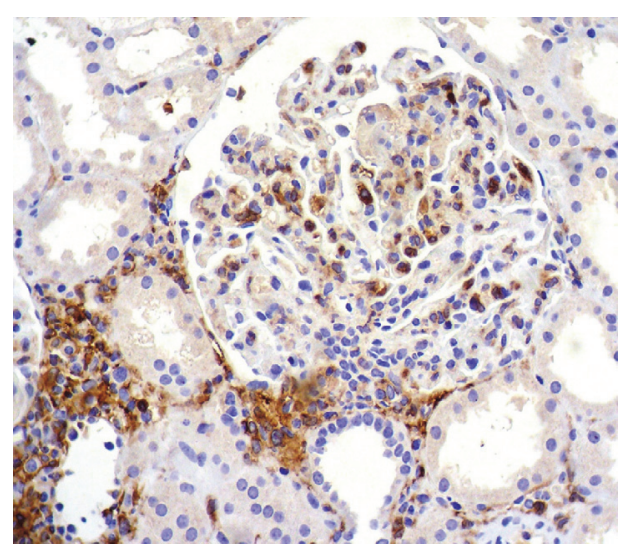

(a)

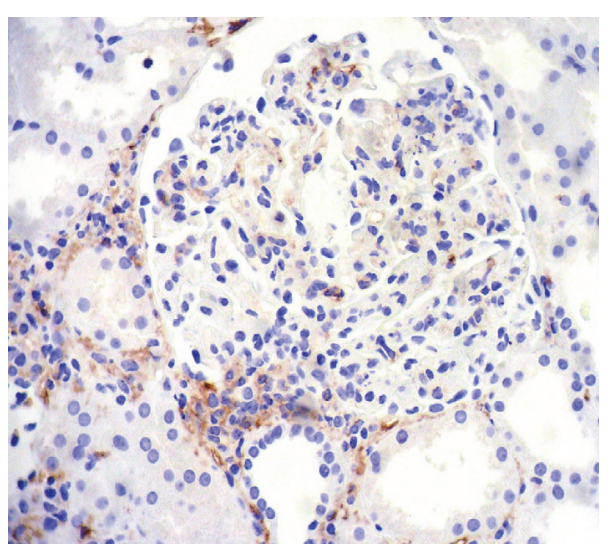

(b)

FIGURE 1: (a) Immunohistochemistry for CD16: abundant $\mathrm{CD}_{16}{ }^{+}$cells in the glomerular tuft; these cells are also evidenced in the interstitium. (b) Immunohistochemistry for CD14: a glomerulus with few positive CD14 ${ }^{+}$cells. There are also some CD14 $4^{+}$cells in the interstitium. Both images, $\times 400$.

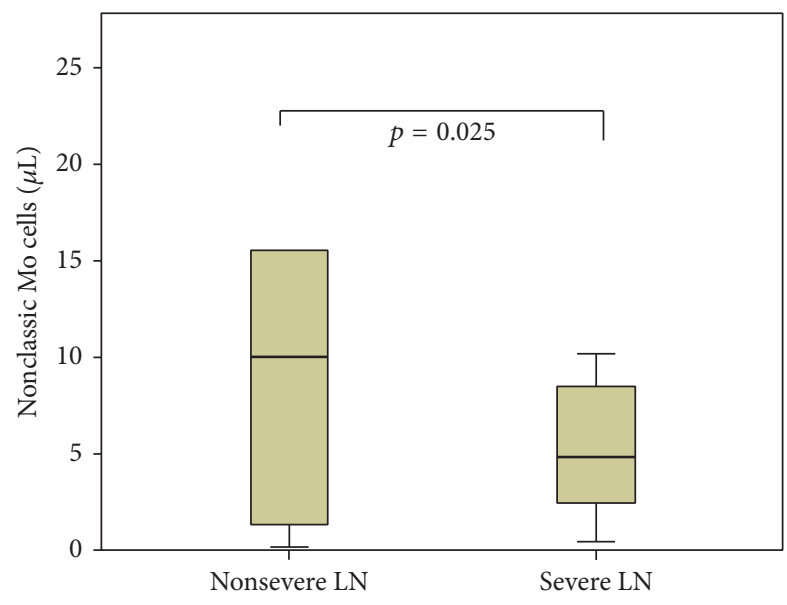

(a)

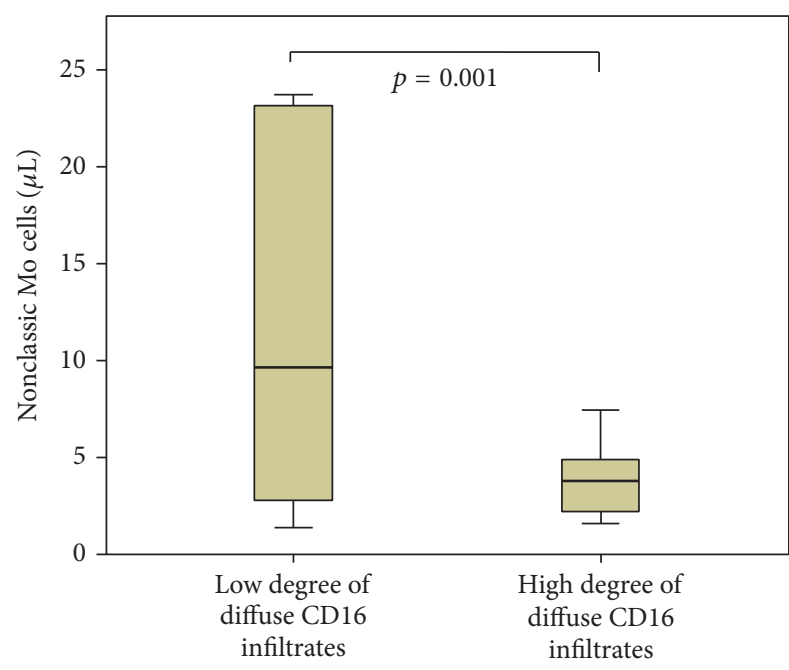

(b)

Figure 2: Peripheral blood levels of Mo subsets according to severity of LN (a) and degree of infiltrations by CD16 ${ }^{+}$cells (b).

accumulated grade 1 aggregates were observed in a patient with Class III LN.

3.3. Peripheral Mo Subsets. Mo subsets around the time of kidney biopsy were available in 21 patients. Mean levels of absolute Mo were $497.63 \pm 329$ cells $/ \mu \mathrm{L}$, classical Mo $459.46 \pm 315.98$ cells $/ \mu \mathrm{L}$, intermediate Mo $17.33 \pm 16.05$ cells $/ \mu \mathrm{L}$, and nonclassic Mo $10.09 \pm 14.82$ cells $/ \mu \mathrm{L}$. When we classified patients in severe and nonsevere forms of $\mathrm{LN}$, severe forms had lower levels of nonclassic Mo in peripheral blood (7.14 \pm 7.22 versus $17.47 \pm 25.32$ cells $/ \mu \mathrm{L}, p=0.025$, Figure 2(a)). When we classified patients according to diffuse $\mathrm{CD}^{+} 6^{+}$infiltrates in high or low degree, patients with higher degree of infiltrates had lower levels of nonclassical Mo in peripheral blood $(37.53 \pm 21.98$ versus $8.39 \pm 5.70$ cells $/ \mu \mathrm{L}$, $p=0.001$, Figure 2(b)). Table 3 summarizes levels of different
Mo subsets according to disease duration, severity of LN, and degree of infiltration.

At the same time, patients with higher degree of $\mathrm{CD} 16^{+}$ infiltrates had higher but not significant activity index scores $(5.46 \pm 4.12$ versus $4.70 \pm 4.71, p=0.363)$ and higher chronicity index scores $(2.0 \pm 2.72$ versus $0.40 \pm 0.51, p=$ 0.004 ). We did not find any association among subsets of Mo in peripheral blood with clinical characteristics, serological markers (including complement antibodies or anti-dsDNA) either with disease activity (SLEDAI).

\section{Discussion}

Mo and macrophages play an important role in murine models and human subjects with renal disease. Those cells have been involved in not only several processes such as 
TABLE 3: Peripheral blood levels of Mo subsets according to disease duration, severity of LN, and degree of infiltrations by CD16 ${ }^{+}$cells.

(a)

\begin{tabular}{lccc}
\hline & Early onset LN & Non-early onset LN & $p$ value \\
\hline Classic $\left(\mathrm{CD} 14^{++} \mathrm{CD} 16^{-}\right)$ & $411.53 \pm 377.48$ & $465.27 \pm 138.48$ & 0.021 \\
Intermediate $\left(\mathrm{CD} 14^{++} \mathrm{CD} 16^{-}\right)$ & $14.35 \pm 15.37$ & $22.41 \pm 18.17$ & 0.488 \\
Nonclassic $\left(\mathrm{CD} 14^{+} \mathrm{CD} 16^{++}\right)$ & $7.51 \pm 7.38$ & $15.32 \pm 20.80$ & 0.138 \\
\hline
\end{tabular}

(b)

\begin{tabular}{lccc}
\hline & Severe LN & Nonsevere LN & $p$ value \\
\hline Classic $\left(\mathrm{CD} 14^{++} \mathrm{CD} 16^{-}\right)$ & $485.80 \pm 345.81$ & $393.63 \pm 239.20$ & 0.340 \\
Intermediate $\left(\mathrm{CD} 14^{++} \mathrm{CD} 16^{-}\right)$ & $18.32 \pm 18.11$ & $14.84 \pm 10.10$ & 0.240 \\
Nonclassic $\left(\mathrm{CD} 14^{+} \mathrm{CD} 16^{++}\right)$ & $7.14 \pm 7.22$ & $17.47 \pm 25.32$ & 0.025 \\
\hline
\end{tabular}

(c)

\begin{tabular}{lccc}
\hline & $\begin{array}{c}\text { High degree of diffuse CD16 } \\
\text { infiltrates }(\geq 5 \text { cells })\end{array}$ & $\begin{array}{c}\text { Low degree of diffuse CD16 } \\
\text { infiltrates }(<5 \text { cells })\end{array}$ & $p$ value \\
\hline Classic $\left(\mathrm{CD} 14^{++} \mathrm{CD} 16^{-}\right)$ & $496.35 \pm 443.38$ & $543.71 \pm 368.67$ & 0.627 \\
Intermediate $\left(\mathrm{CD} 14^{++} \mathrm{CD} 16^{-}\right)$ & $9.65 \pm 7.35$ & $17.06 \pm 18.79$ & 0.198 \\
Nonclassic $\left(\mathrm{CD} 14^{+} \mathrm{CD} 16^{++}\right)$ & $3.95 \pm 2.32$ & $11.70 \pm 10.37$ & 0.001 \\
\hline
\end{tabular}

Severe LN was defined as either Class III or Class IV LN.

renal injury and fibrosis but also several repair processes. We found that patients with more severe forms of LN (proliferative forms) have a higher grade of diffuse $\mathrm{CD} 16^{+}$ Mo infiltrates. At the same time, severe forms had lower levels of peripheral nonclassical Mo and might reflect organ recruitment phenomenon of Mo cells in more severe forms of LN (proliferative Classes III and IV).

Linage cells from $\mathrm{Mo} / \mathrm{macrophages} \mathrm{system} \mathrm{constitute} \mathrm{one}$ of the most important cells involved in the inflammatory process of several renal diseases. Several studies have shown that the amounts of macrophages found in damaged tissues not only are bystanders of the inflammatory process but also play a role as active cells as occurs in some other in vitro studies [16]. Macrophages are distributed in tissues throughout the body and contribute to both homeostasis and disease. The recent increase in the availability of diverse surface markers for the determination of $\mathrm{Mo} /$ macrophages allowed the identification of different subpopulations in the kidney and other tissues. Mo subpopulations are functionally different. Some of these subpopulations have been related with a selective pattern of cytokine production $[7,8]$.

In vitro studies have demonstrated that macrophages can be polarized by activation with several cytokines. Activated and polarized cells have different functions. Initially, polarized cells were known as "classic" or "alternative" Mo. However, some other authors suggest that Mo should be labeled according to their phenotype (based on surface molecules) or according to their function [17-19]. The difference in the expression of lipopolysaccharide (LPS) receptor (CD14) and of $\mathrm{F} c \gamma$ receptor III (CD16) has been used to distinguish various Mo subpopulations [4, 20].

We found a greater amount of diffuse infiltrations and aggregates of $\mathrm{CD}^{+} 6^{+}$cells in patients with more severe forms of $\mathrm{LN} . \mathrm{CD}^{+} 6^{+}$are considered as nonclassic or intermediate Mo. It is well known that $\mathrm{CD}_{1}{ }^{+}$cells produce TNF- $\alpha$ and interferon gamma (IFN $\gamma$ ) and participate in inflammatory process. Additionally, $\mathrm{CD}^{+} 6^{+}$express Class II molecules with a more efficient capacity for antigen presentation.

Previously, we observed that patients suffering from SLE had lower levels of $\mathrm{CD}^{+} 6^{+}$in peripheral blood, suggesting a differentiation process out of organs and cell recruitment in damaged organs [6]. Mo recruitment in inflammatory tissues could be explained in part by the active participation of Mo during all stages of organ damage process due to autoimmune phenomena. Mo participate during the clearance process of apoptotic bodies and immune complexes. In addition, Mo can induce the production of several cytokines involved in the pathogenesis of LN (for instance, IFN $\gamma$ ). Finally, Mo promote fibrosis and loss of epithelial cells and microvasculature [21, $22]$. Given the association between chronicity index score and high degree of $\mathrm{CD}^{+} 6^{+}$infiltrates in our study, it is possible that CD16 Mo participate not only during early acute glomerular damage process but also during chronic fibrotic and microvascular processes as we mentioned previously.

Fractalkine (CX3CL1) and its receptor, CX3CR1, are known to mediate both cell adhesion and cell migration. In humans, CX3CR1 is expressed on Mo/macrophages and cytotoxic effector lymphocytes such as natural killer cells and cytotoxic $\mathrm{T}$ cells. In mice, its expression in circulating blood cells is restricted to Mo/macrophages and NK cells. Murine and human studies focused on the role of Mo in LN are limited. Using MRL/lpr murine model, Nakatani and colleagues [11] showed that fractalkine expression and $\mathrm{CD}_{16}{ }^{+}$Mo infiltration increase with progression of the LN. In another study, a fractalkine antagonist was administered to MRL/lpr mice during early stages of LN [23]. MRL/lpr 
mice that received fractalkine antagonist exhibited significantly reduced glomerular hypercellularity, glomerulosclerosis, crescent formation, and vasculitis when compared with control mice.

Yoshimoto et al. [12] evaluated glomerular expression of fractalkine and $\mathrm{CD} 16^{+} \mathrm{Mo}$ in patients with LN. The authors found correlation of fractalkine glomerular expression with histopathologic activity index and proliferative forms of LN. Additionally, the amounts of infiltrating $\mathrm{CD}^{+} 6^{+}$Mo were higher in proliferative glomerular lesions of patients with SLE.

To the best our knowledge, this is the first study that correlated the levels of Mo in peripheral blood with Mo infiltrates in renal tissues. Our study suggested an inverse correlation among peripheral levels of nonclassic Mo and higher diffuse $\mathrm{CD}^{+} 6^{+}$infiltrative cells in glomerular tissue in patients with proliferative forms of LN.

Our study has some limitations. First, we analyzed a relative small number of patients with biopsy proven LN. However, despite this small sample we found significant differences among groups (severe and nonsevere) according to $\mathrm{CD}_{16}{ }^{+}$infiltrates and subsets of Mo in peripheral blood. Given the nature of the study, we were not able to measure at the same time subsets of Mo in peripheral blood in all patients tested for Mo infiltrates on renal samples. Likewise, as our hospital is a reference center for systemic autoimmune diseases and predominantly received patients with limited access to health care, therefore our patients could be a selected sample of more severe SLE patients with LN. Finally, given that the majority of our patients were Mestizo, our results must be interpreted carefully and must be confirmed in other populations with different racial/ethnic distributions.

In conclusion our results in Mestizo patients with biopsy proven LN suggest that those patients with more severe forms of LN had lower peripheral levels of nonclassical Mo $\left(\mathrm{CD} 14^{+} \mathrm{CD}_{16}{ }^{++}\right)$. Reduction of these Mo subsets could be explained in part by a recruitment process in renal tissues. Given the small sample of the current study, our results must be interpreted carefully. Although the information about the role of Mo in LN is limited in clinical studies, animal model studies using fractalkine antagonist open a potential new target for the treatment of patients suffering from severe forms of LN. So far, there are no ongoing clinical trials evaluating the potential benefit of fractalkine antagonist in patients with LN.

\section{Abbreviations}

DC: Dendritic cells

IFN $\gamma$ : Interferon gamma

LN: $\quad$ Lupus nephritis

LPS: Lipopolysaccharide

Mo: $\quad$ Monocytes

SLE: Systemic lupus erythematosus

SLEDAI: Systemic Lupus Erythematosus Disease Activity Index.

\section{Competing Interests}

The authors declare that they have no competing interests.

\section{Authors' Contributions}

Anabel Barrera García conceived the study, participated in data analysis, and approved the final version of the manuscript. José A. Gómez-Puerta participated in its design and wrote and approved the final version of the manuscript. Luis F. Arias participated in data analysis of kidney biopsies and approved the final version of the manuscript. Catalina Burbano and Mauricio Rojas participated in data analysis of monocytes subsets and reviewed and approved the final version of the manuscript. Mauricio Restrepo, Adriana L. Vanegas, Carlos H. Muñoz, and Luis A. González participated in patient recruitment and reviewed and approved the final version of the manuscript. Gloria Vásquez participated in the design of the study, supported data analysis, and reviewed and approved the final version of the manuscript. All authors read and approved the final manuscript.

\section{Acknowledgments}

This work was supported by Colciencias Project no. 11556933532 (Contract no. 575-2013) and by Programa de Sostenibilidad, Universidad de Antioquia. J. A. GómezPuerta was supported by Colciencias (no. 656/2014).

\section{References}

[1] L. Lisnevskaia, G. Murphy, and D. Isenberg, "Systemic lupus erythematosus," The Lancet, vol. 384, no. 9957, pp. 1878-1888, 2014.

[2] M. Adler, S. Chambers, C. Edwards, G. Neild, and D. Isenberg, "An assessment of renal failure in an SLE cohort with special reference to ethnicity, over a 25 -year period," Rheumatology, vol. 45, no. 9, pp. 1144-1147, 2006.

[3] J. A. Gómez-Puerta, C. H. Feldman, G. S. Alarcón, H. Guan, W. C. Winkelmayer, and K. H. Costenbader, "Racial and ethnic differences in mortality and cardiovascular events among patients with End-stage renal disease due to lupus nephritis," Arthritis Care \& Research, vol. 67, no. 10, pp. 1453-1462, 2015.

[4] E. Grage-Griebenow, H.-D. Flad, and M. Ernst, "Heterogeneity of human peripheral blood monocyte subsets," Journal of Leukocyte Biology, vol. 69, no. 1, pp. 11-20, 2001.

[5] L. Ziegler-Heitbrock, "The CD14+ CD16+ blood monocytes: their role in infection and inflammation," Journal of Leukocyte Biology, vol. 81, no. 3, pp. 584-592, 2007.

[6] C. Burbano, G. Vasquez, and M. Rojas, "Modulatory effects of cd14+cd16++ monocytes on cd14++cd16- monocytes: a possible explanation of monocyte alterations in systemic lupus erythematosus," Arthritis and Rheumatology, vol. 66, no. 12, pp. 3371-3381, 2014.

[7] K.-U. Belge, F. Dayyani, A. Horelt et al., “The proinflammatory $\mathrm{CD} 14^{+} \mathrm{CD} 16^{+} \mathrm{DR}^{++}$monocytes are a major source of TNF," Journal of Immunology, vol. 168, no. 7, pp. 3536-3542, 2002.

[8] J. Skrzeczyńska-Moncznik, M. Bzowska, S. Loseke, E. GrageGriebenow, M. Zembala, and J. Pryjma, "Peripheral blood CD14 ${ }^{\text {high }} \mathrm{CD}^{+} 6^{+}$monocytes are main producers of IL-10," Scandinavian Journal of Immunology, vol. 67, no. 2, pp. 152-159, 2008.

[9] D. Strauss-Ayali, S. M. Conrad, and D. M. Mosser, "Monocyte subpopulations and their differentiation patterns during 
infection," Journal of Leukocyte Biology, vol. 82, no. 2, pp. 244252, 2007.

[10] D. M. Mosser and J. P. Edwards, "Exploring the full spectrum of macrophage activation," Nature Reviews Immunology, vol. 8, no. 12, pp. 958-969, 2008.

[11] K. Nakatani, S. Yoshimoto, M. Iwano et al., "Fractalkine expression and CD16+ monocyte accumulation in glomerular lesions: association with their severity and diversity in lupus models," American Journal of Physiology-Renal Physiology, vol. 299, no. 1, pp. F207-F216, 2010.

[12] S. Yoshimoto, K. Nakatani, M. Iwano et al., "Elevated levels of fractalkine expression and accumulation of $\mathrm{CD}^{+} 6^{+}$monocytes in glomeruli of active lupus nephritis," American Journal of Kidney Diseases, vol. 50, no. 1, pp. 47-58, 2007.

[13] Y. Li, P. Y. Lee, E. S. Sobel et al., "Increased expression of Fc $\gamma$ RI/CD64 on circulating monocytes parallels ongoing inflammation and nephritis in lupus," Arthritis Research \& Therapy, vol. 11, no. 1, article R6, 2009.

[14] B. H. Rovin, "The chemokine network in systemic lupus erythematous nephritis," Frontiers in Bioscience, vol. 13, no. 3, pp. 904-922, 2008.

[15] I. Heimbeck, T. P. J. Hofer, C. Eder et al., "Standardized singleplatform assay for human monocyte subpopulations: lower $\mathrm{CD} 14^{+} \mathrm{CD} 16^{++}$monocytes in females," Cytometry Part A, vol. 77, no. 9, pp. 823-830, 2010.

[16] J. S. Duffield, "Macrophages and immunologic inflammation of the kidney," Seminars in Nephrology, vol. 30, no. 3, pp. 234-254, 2010.

[17] S. Goerdt and C. E. Orfanos, "Other functions, other genes: alternative activation of antigen-presenting cells," Immunity, vol. 10, no. 2, pp. 137-142, 1999.

[18] V. A. Fadok, D. L. Bratton, A. Konowal, P. W. Freed, J. Y. Westcott, and P. M. Henson, "Macrophages that have ingested apoptotic cells in vitro inhibit proinflammatory cytokine production through autocrine/paracrine mechanisms involving TGF- $\beta$, PGE2, and PAF," Journal of Clinical Investigation, vol. 101, no. 4, pp. 890-898, 1998.

[19] A. Mantovani, A. Sica, and M. Locati, "Macrophage polarization comes of age," Immunity, vol. 23, no. 4, pp. 344-346, 2005.

[20] H. W. L. Ziegler-Heitbrock, "Heterogeneity of human blood monocytes: the $\mathrm{CD}_{1} 4^{+} \mathrm{CD} 16^{+}$subpopulation," Immunology Today, vol. 17, no. 9, pp. 424-428, 1996.

[21] J. S. Duffield, L.-P. Erwig, X.-Q. Wei, F. Y. Liew, A. J. Rees, and J. S. Savill, "Activated macrophages direct apoptosis and suppress mitosis of mesangial cells," The Journal of Immunology, vol. 164, no. 4, pp. 2110-2119, 2000.

[22] J. S. Duffield, P. G. Tipping, T. Kipari et al., "Conditional ablation of macrophages halts progression of crescentic glomerulonephritis," The American Journal of Pathology, vol. 167, no. 5, pp. 1207-1219, 2005.

[23] A. Inoue, H. Hasegawa, M. Kohno et al., "Antagonist of fractalkine (CX3CL1) delays the initiation and ameliorates the progression of lupus nephritis in MRL/lpr mice," Arthritis and Rheumatism, vol. 52, no. 5, pp. 1522-1533, 2005. 


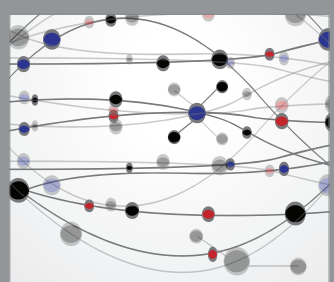

The Scientific World Journal
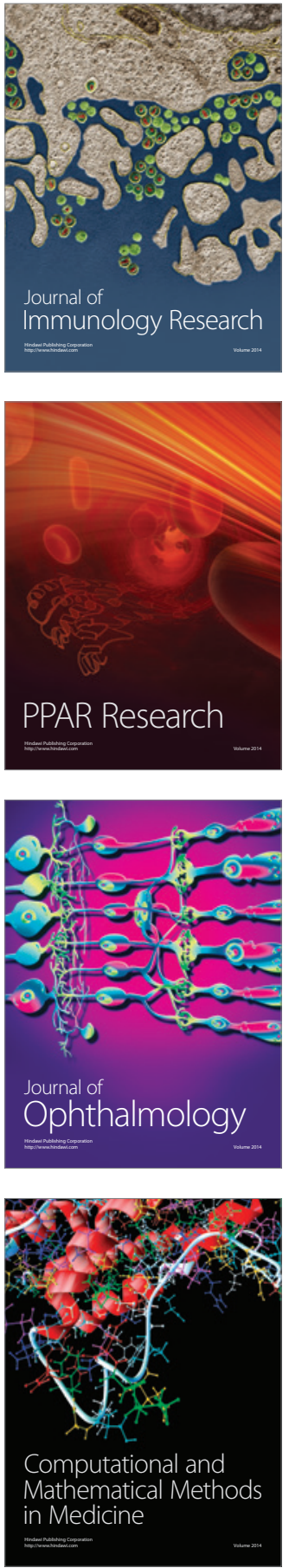

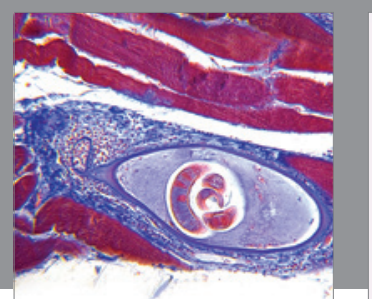

Gastroenterology Research and Practice

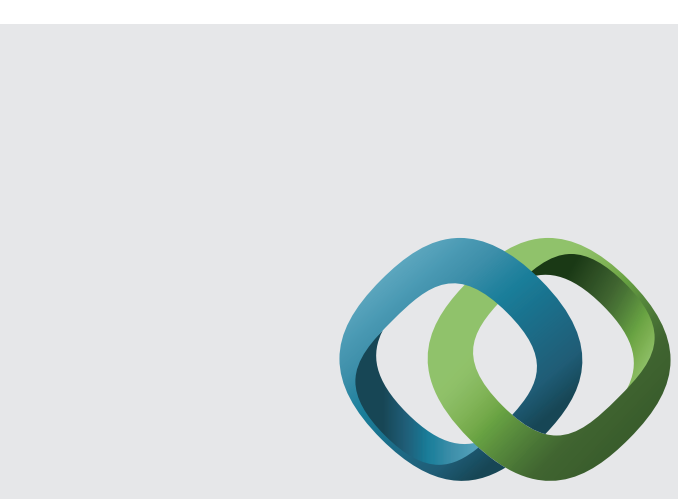

\section{Hindawi}

Submit your manuscripts at

http://www.hindawi.com
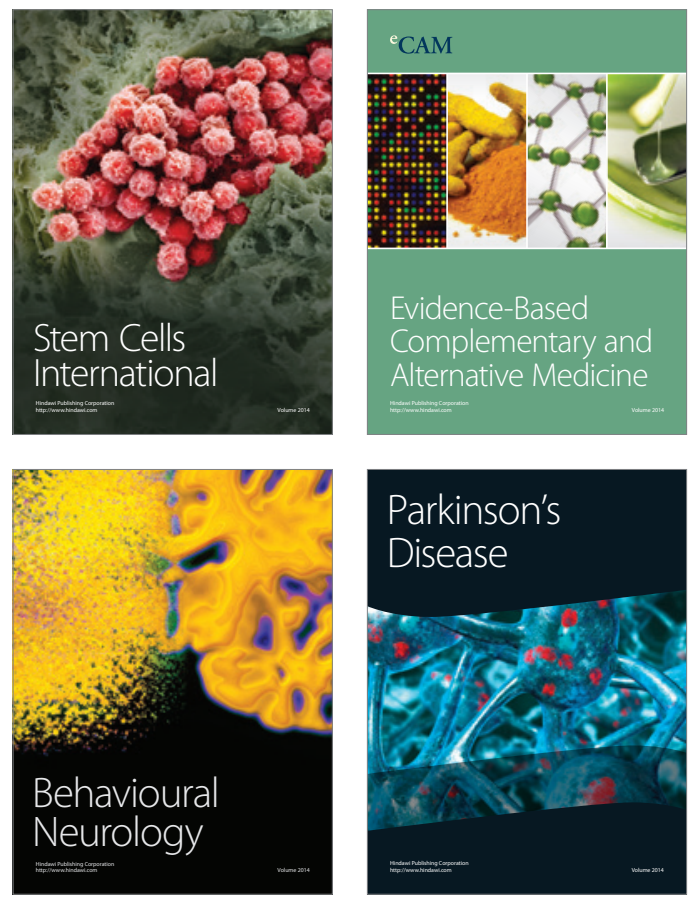
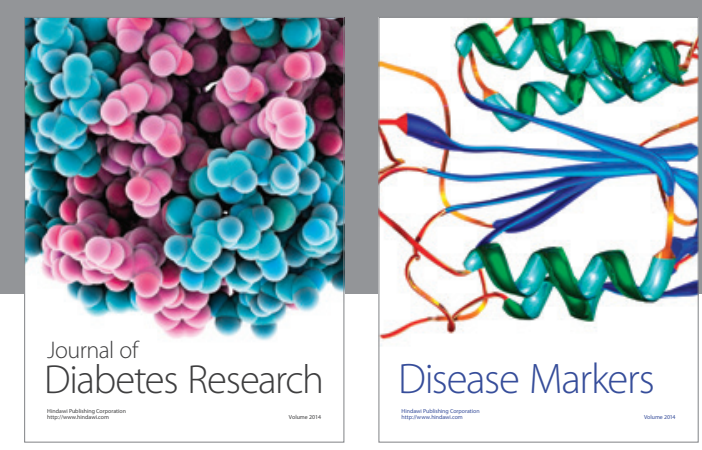

Disease Markers
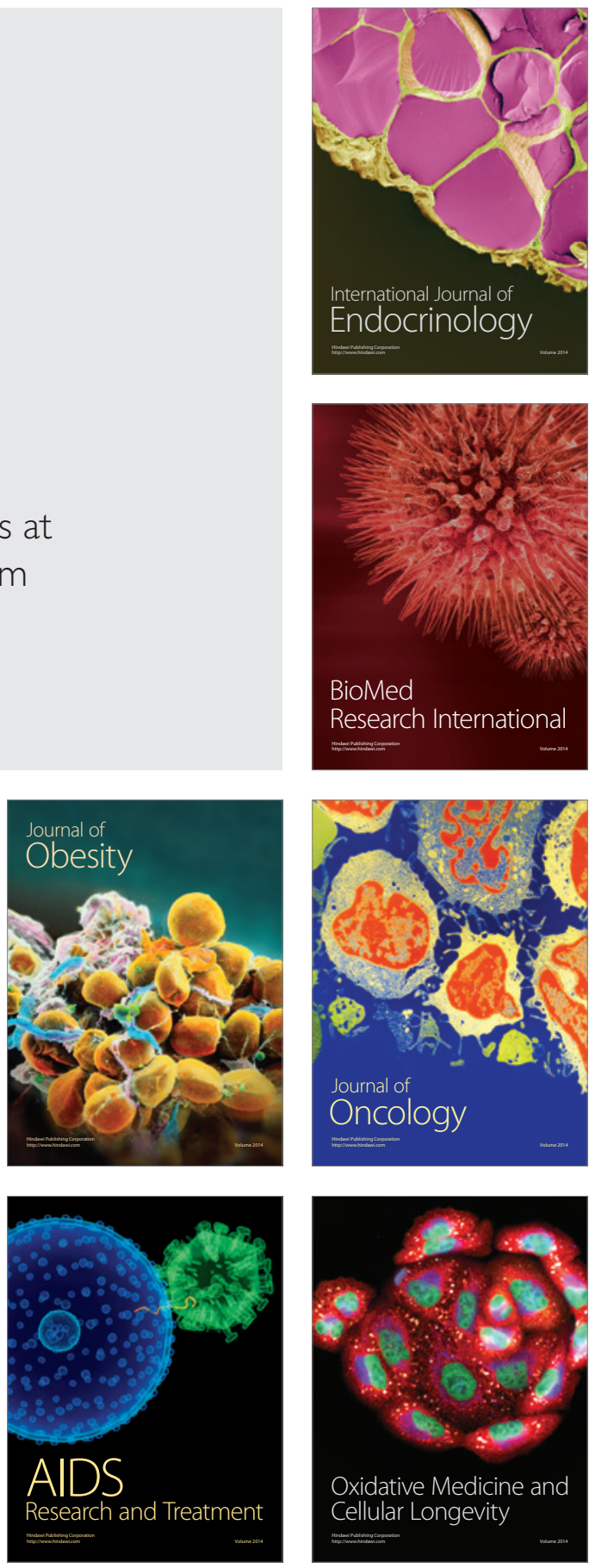